\title{
SOME COMMENTS ON THE STRUCTURE OF COMPACT DECOMPOSITIONS OF $S^{3}$
}

\author{
H. W. LAMBERT
}

In this note we derive some theorems of a general nature on compact, upper semicontinuous decompositions of $S^{3}$, spherical 3-space. A compact decomposition $G$ of $S^{3}$ is one obtained from a compact, proper subset $D$ of $S^{3}$ by setting $G=\{g: g$ is either a component of $D$ or a point of $\left.S^{3}-D\right\}$. In this paper a point-like, compact decomposition of $S^{3}$ is one such that the complement of each component of $D$ is homeomorphic to $E^{3}$, Euclidean 3-space; and a 1-dimensional, compact decomposition of $S^{3}$ is one such that each component of $D$ has dimension no greater than 1 .

Theorem 1 shows that in the decomposition space of a point-like, compact decomposition of $S^{3}$ the collection of points which fail to have some neighborhood homeomorphic to $E^{3}$ is dense in itself. The remaining theorems are concerned with the effect of inserting or removing certain elements from a compact decomposition of $S^{3}$. Armentrout's [2, Theorems 1,6] are essential to our investigation. A version of these theorems appears below as Theorem A. A summary of some known results on compact decompositions of $S^{3}$ may be found in [3].

We assume then that $G$ denotes a compact decomposition of $S^{3}$ with associated projection map $P$ onto the decomposition space $S^{3} / G$. Let $H_{G}$ denote the sum of the nondegenerate elements of $G$. Note that $P\left(\mathrm{Cl} H_{G}\right)$ is compact and 0 -dimensional. It is known that there is a sequence of compact polyhedral 3-manifolds with boundary in $S^{3}$ such that $\bigcap_{i=1}^{\infty} M_{i}=\mathrm{Cl} H_{G}$ and, for each $i, M_{i+1} \subset$ Int $M_{i}$. Such a sequence $M_{i}$ will be referred to as a defining sequence for $G$. Let $Q=\left\{x: x \in S^{3} / G\right.$ and $x$ has a neighborhood homeomorphic to $\left.E^{3}\right\}$, and let $F=S^{3} / G-Q$. Note that if $F \neq \varnothing$, then $F$ is compact and 0 -dimensional.

TheOrem A. Assume $G$ is a compact decomposition of $S^{3}\left(P\left(\mathrm{Cl} H_{G}\right)\right.$ is compact and 0-dimensional). Let $M$ be a compact polyhedral 3-manifold with boundary in $S^{3}$ such that $\mathrm{Bd} M \cap C l H_{G}=\varnothing$. If $P(M) \subset Q$ and either $G$ is a point-like decomposition or $M$ has a triangulation whose 1-skeleton is disjoint from $\mathrm{Cl} H_{G}$, then there is a map of $M$ onto itself fixed on $\mathrm{Bd} M$ and inducing the same decomposition as $G$ restricted to $M$.

Received by the editors October 12, 1966. 
Proof. Since either $G$ is a point-like decomposition or $M$ has a triangulation whose 1 -skeleton is disjoint from $\mathrm{Cl} H_{G}$, it follows that there is a collection of disjoint polyhedral arcs $A_{1}, \cdots, A_{n}$ such that each Int $A_{i} \subset$ Int $M-\mathrm{Cl} H_{G}$, each $\mathrm{Bd} A_{i} \subset \mathrm{Bd} M$, and any two boundary components of any component of $M$ are connected by one of the $A_{i}$. For each $i$, let $A_{i}^{\prime}$ be an open regular neighborhood of $A_{i}$ in $M$ such that $\mathrm{Cl} A_{i}^{\prime} \cap \mathrm{Cl} A_{j}^{\prime}=\varnothing$ for $i \neq j$ and each $\mathrm{Cl} A_{i}^{\prime}$ $\cap \mathrm{Cl} H_{G}=\varnothing$. Let $M^{\prime}=M-\cup_{i=1}^{n} A_{i}^{\prime}$. Since the boundary of each component of $M^{\prime}$ is connected, it follows from the proof of either [2, Theorem 1] (if $G$ is point-like) or [2, Theorem 6] (if $M$ has a triangulation whose 1-skeleton is disjoint from $\mathrm{Cl} H_{G}$ ) that there is a homeomorphism $h^{\prime}$ of $M^{\prime}$ onto $P\left(M^{\prime}\right)$ such that $h^{\prime} \mid \mathrm{Bd} M^{\prime}$ $=P \mid \operatorname{Bd} M^{\prime}$. Extending $h^{\prime}$ by using $P$ on each $A_{i}^{\prime}$, we see that there is a homeomorphism $h$ of $M$ onto $P(M)$ such that $h|\operatorname{Bd} M=P| \operatorname{Bd} M$. The required map is $h^{-1} P$.

TheOREM 1. Let $G$ be a point-like, compact decomposition of $S^{3}$. Then $F$ is either empty or a Cantor set, that is F has no isolated points.

Proof. Suppose that $F$ has an isolated point $x$. In the defining sequence $M_{i}$ of $G$, let $n$ be chosen large enough that the component $K$ of $M_{n}$ containing $P^{-1}(x)$ is such that $P(K)-x \subset Q$. Since $G$ is pointlike there is a map $f^{\prime}$ of $K$ onto itself such that $f^{\prime}$ is fixed on $\operatorname{Bd} K$, $f^{\prime}$ is a homeomorphism on $K-P^{-1}(x)$ and $f^{\prime}\left(P^{-1}(x)\right)$ is a point. For $i=1,2,3, \cdots$, let $K_{i}=M_{n+i} \cap K$ and let $L_{i}$ be the component of $K_{i}$ containing $P^{-1}(x)$. Let $K_{1}^{\prime}=K_{1}-L_{1}$, and, for each $i$, let $K_{i+1}^{\prime}=K_{i+1}$ $-\bigcup_{j=1}^{i}\left(K_{j}^{\prime}\right) \cup L_{i+1}$. Since each $P\left(K_{i}^{\prime}\right) \subset Q$, it follows from Theorem A that there is a mapping $f_{i}$ of $K_{i}^{\prime}$ on to itself fixed on $\mathrm{Bd} K_{i}^{\prime}$ and inducing the same decomposition as $G$ restricted to $K_{i}^{\prime}$. Assuming each $f_{i}=$ identity on $K-K_{i}^{\prime}$, we have that $f=f^{\prime}\left(\prod_{i=1}^{\infty} f_{i}\right)$ is a map of $K$ onto itself fixed on Bd $K$ and inducing the same decomposition as $G$ restricted to $K$. It follows that the map $P f^{-1}$ is a homeomorphism of $K$ on to $P(K)$. But this contradicts that $x \in F$. Therefore $F$ is either empty or it is homeomorphic to a Cantor set.

In [6], Finney showed that if $f$ is a point-like, simplicial map of $S^{3}$, then $f\left(S^{3}\right)$ is homeomorphic to $S^{3}$. To prove this he simplified the decomposition $G_{f}$ of $S^{3}$ induced by $f$. This simplification was accomplished by a process of deleting portions of certain nondegenerate elements of $G_{f}$ to obtain a new decomposition $G_{f}^{\prime}$ such that $S^{3} / G_{f}^{\prime}$ is homeomorphic to $S^{3} / G_{f}$. In Theorems 2 and 3 it is shown that, for compact decompositions $G$ of $S^{3}$, we may delete certain elements of $G$ to form a new decomposition $G^{\prime}$ such that $S^{3} / G$ is homeomorphic to $S^{3} / G^{\prime}$. 
THEOREM 2. Let $G$ be a point-like, compact decomposition of $S^{3}$. Let $U$ be an open set in $S^{3}$ such that $U$ is the union of elements in $G$ and $P(U) \subset Q$. Let $G^{\prime}$ be the decomposition obtained by points of $U$ and elements of $G$ in $S^{3}-U$. Then $S^{3} / G$ is homeomorphic to $S^{3} / G^{\prime}$.

Proof. It is easily checked that $G^{\prime}$ is an upper semicontinuous decomposition of $S^{3}$. Let $P^{\prime}$ be the projection map for $G^{\prime}$. Let $M_{i}$ be a defining sequence for $G$. Define $K_{i}$ to be the union of all components of $M_{i}$ which do not intersect $S^{3}-U$. Let $K_{1}^{\prime}=K_{1}$ and, for $i=1,2, \cdots$, let $K_{i+1}^{\prime}=K_{i+1}-\bigcup_{j=1}^{i} K_{j}^{\prime}$. Since $P\left(K_{i}^{\prime}\right) \subset Q$ it follows by Theorem A that there is a map $f_{i}$ of $S^{3}$ onto itself fixed on $S^{3}-\operatorname{Int} K_{i}^{\prime}$ and inducing the same decomposition as $G$ restricted to $K_{i}^{\prime}$. Let $f=\prod_{i-1}^{\infty} f_{i}$. Then $P^{\prime} f P^{-1}$ is a 1-1 correspondence between the points of $S^{3} / G$ and $S^{3} / G^{\prime}$. If $K$ is a component of some $K_{i}^{\prime}$, then $f^{-1}(K)=K$. Using this fact and that $f$ is a continuous map on $U$ that induces the same decomposition as $G$ restricted to $U$, it follows that if $V$ is open in $S^{3}$ and $V$ is the union of elements in $G^{\prime}$, then $f^{-1}(V)$ is open in $S^{3}$ and $P\left(f^{-1}(V)\right)$ is open in $S^{3} / G$. Hence $P^{\prime} f P^{-1}$ is a continuous 1-1 map of $S^{3} / G$ onto $S^{3} / G^{\prime}$, and it follows that $P^{\prime} f P^{-1}$ is a homeomorphism between these spaces.

Theorem 3 is similar to Theorem 2, except that we consider 1dimensional, compact decompositions of $S^{3}$ instead of point-like, compact decompositions of $S^{3}$. To prove Theorem 3 the following lemma is needed. A version of this lemma was proved independently by Alford and Sher in [1], but our proof differs from theirs in that we do not use Kwun and Raymond's [7, Theorem 3, Corollary 2].

Lemma. Let $G$ be a 1-dimensional, compact decomposition of $S^{3}$ and let $M$ be a compact polyhedral 3-manifold with boundary in $S^{3}$ such that $\mathrm{Bd} M \cap \mathrm{Cl} H_{G}=\varnothing, P(M) \subset Q$, and $P(M)$ is embeddable in $S^{3}$. Then there is a map of $M$ onto itself fixed on $\mathrm{Bd} M$ and inducing the same decomposition as $G$ restricted to $M$.

Proof. Let $T$ be a triangulation of $M$ such that each 1-simplex of $T$ that intersects $\mathrm{Bd} M$ is disjoint from $\mathrm{Cl} H_{G} \cap M$. Since $\operatorname{dim} g \leqq 1$ for each $g \in G$, we may assume that the 0 -skeleton of $T$ is disjoint from $\mathrm{Cl} H_{G}$. Let $A$ be a 1-simplex of $T$ with endpoints $a$ and $b$ such that $A \cap \mathrm{Bd} M=\varnothing$, and let $K$ be a polyhedral cube in Int $M$ obtained by thickening Int $A$ slightly so that $\mathrm{Bd} A \subset \mathrm{Bd} K$ and $K \cap T_{1}=A$, where $T_{1}$ is the carrier of the 1 -skeleton of $T$. Let $U$ be a complementary domain of $\mathrm{Bd} K$. Since $\operatorname{dim} g \leqq 1$ for each $g \in G$ and $P\left(\mathrm{Cl} H_{G}\right)$ is 0 dimensional, it follows that $U-\mathrm{Cl} H_{G}$ is connected. Hence there is an $\operatorname{arc} X$ with endpoints $a$ and $b$ such that Int $X \subset U$ and $X \cap C l H_{G}$ 
$=\varnothing$. Similarly there is an arc $Y$ with endpoints $a$ and $b$ such that Int $Y \subset\left(S^{3}-\mathrm{Cl} U\right)$ and $Y \cap C l H_{G}=\varnothing$. Since $\mathrm{Bd} M \cap \mathrm{Cl} H_{G}=\varnothing$, we may assume $X \cup Y \subset$ Int $M$.

Suppose Bd $K \cap \mathrm{Cl} H_{G}$ separates $a$ from $b$ on Bd $K$. Let $S$ be the component of $\mathrm{Bd} K-\mathrm{Cl} H_{G}$ containing $a$. It follows that $P(\mathrm{Cl} S)$ is a singular 2-sphere in Int $P(M)$ (regard $P(\mathrm{Cl} S)$ as the image of Bd $K$ under the map $g(x)=P(x)$ if $x \in S$ and $g(x)=P(\operatorname{Bd} C)$ if $x$ belongs to the component $C$ of $\mathrm{Bd} K-S$ ) and that the simple closed curve $P(X \cup Y)$ intersects and pierces $P(\mathrm{Cl} S)$ at just one point. But this is impossible since $P(M)$ is embeddable in $S^{3}$. Hence there is an $\operatorname{arc} B$ on Bd $K$ with endpoints $a$ and $b$ such that $B \cap C l H_{G}=\varnothing$. There is a homeomorphism of $M$ onto itself which is fixed on ( $\mathrm{Bd} M \cup T_{1}$ ) - Int $A$ and takes $A$ onto $B$. Repeating this argument a finite number of times, we push $T_{1}$ off $\mathrm{Cl} H_{G}$. Since $P(M) \subset Q$, this lemma now follows from Theorem A.

Theorem 3. Let $G$ be a 1-dimensional, compact decomposition of $S^{3}$ and let $U$ be an open set in $S^{3}$ such that $U$ is the union of elements in $G$ and $P(U) \subset Q$. Let $G^{\prime}$ be the decomposition obtained by points of $U$ and elements of $G$ in $S^{3}-U$. Then $S^{3} / G$ is homeomorphic to $S^{3} / G^{\prime}$.

Proof. As in Theorem 2 we may choose a sequence $K_{i}^{\prime}$ of disjoint 3-manifolds in $U$ such that $U \cap \mathrm{Cl} H_{G} \subset \bigcup_{i=1}^{\infty} K_{i}^{\prime}$. Each $K_{i}^{\prime}$ can be chosen so that $P\left(K_{i}^{\prime}\right)$ is embeddable in $S^{3}$. By the previous lemma there is a map of $S^{3}$ onto itself fixed on $S^{3}-$ Int $K_{i}^{\prime}$ and inducing the same decomposition as $G$ restricted to $K_{i}^{\prime}$. The remainder of this proof is the same as the proof of Theorem 2 .

CorollaRY. Let $G$ be a 1-dimensional, compact decomposition of $S^{3}$ such that $S^{3} / G$ is a 3-manifold. Then $S^{3} / G$ is homeomorphic to $S^{3}$.

As pointed out by Bing in $\left[4\right.$, p. 7], it may be shown that if $C_{1}$, $C_{2}, \cdots, C_{n}$ are mutually exclusive, nonseparating continua in $S^{3}$, then there is a decomposition $G$ of $S^{3}$ such that each $C_{i} \in G$ and $S^{3} / G$ is homeomorphic to $S^{3}$. The next theorem shows that while staying in the category of point-like, compact decompositions we cannot change an element of $G$ whose image is in $F$ to one whose image is in $Q$ by adding more nondegenerate elements to $G$.

Theorem 4. Let $G$ and $G^{\prime}$ be point-like, compact decompositions of $S^{3}$ such that each nondegenerate element of $G$ is contained in $G^{\prime}$. If $g \in G$ and $P(g) \in F$, then $P^{\prime}(g) \in F^{\prime}$ (where $P^{\prime}$ is the projection map associated with $G^{\prime}$ and $F^{\prime}$ is the non-Euclidean points of $\left.S^{3} / G^{\prime}\right)$. 
Proof. Assume by way of contradiction that $g_{0}$ is an element of $G$ such that $P\left(g_{0}\right) \in F$ but $P^{\prime}\left(g_{0}\right) \in Q^{\prime}$ (where $Q^{\prime}=S^{3} / G^{\prime}-F^{\prime}$ ). Let $M_{i}$ and $M_{i}^{\prime}$ be defining sequences for $G$ and $G^{\prime}$, respectively. Let $K$ be a component of some $M_{n}$ such that $g_{0} \subset K, P^{\prime}(K) \subset Q^{\prime}$, and, for each $i=1,2, \cdots$, let $K_{i}=K \cap M_{n+i}$. If for each positive integer $m$ and each positive number $\epsilon$ there is a homeomorphism $h$ of $S^{3}$ on to itself such that: (1) if $x \in S^{3}-K_{m}$, then $h(x)=x$; and (2) if $g \in G$ and $g \subset K_{m}$, then diam $h(g)<\epsilon$, then it follows from the proof of Theorem 1 of [5] that $P(K)$ is homeomorphic to $K$. This would contradict that $P\left(g_{0}\right) \in F$ and establish the theorem.

Hence let $m$ and $\epsilon$ be given. Let $K^{\prime}$ be the union of all elements of $G$ contained in $K$ and of diameter greater than or equal to $\epsilon$. There is a positive integer $p$ such that the components of $M_{p}^{\prime}$ which intersect $K^{\prime}$ are contained in $K_{m}$. Denote the union of these components by $S$. Since $P^{\prime}(S) \subset Q^{\prime}$, it follows from the proof of Theorem 2 of [2] that there is a homeomorphism $h$ of $S^{3}$ onto itself such that: (1) if $x \in S^{3}-S$, then $h(x)=x$; and (2) if $g^{\prime} \in G^{\prime}$ and $g^{\prime} \subset S$, then diam $h\left(g^{\prime}\right)$ $<\epsilon$. This homeomorphism satisfies the desired properties given in the previous paragraph and completes the proof.

\section{REFERENCES}

1. W. R. Alford and R. B. Sher, Some decompositions of 3-space that are factors of 4-space, Amer. Math. Monthly (to appear).

2. S. Armentrout, Decompositions of $E^{3}$ with a compact 0 -dimensional set of nondegenerate elements, Trans. Amer. Math. Soc. 123 (1966), 165-177.

3. - Monotone decompositions of $E^{3}$, Annals of Mathematics Studies, No. 60, Princeton Univ. Press, Princeton, N.J., 1966.

4. R. H. Bing, "Decompositions of $E^{3}$," in Topology of 3-manifolds and related topics, pp. 5-21, Prentice-Hall, Englewood Cliffs, N. J., 1962.

5. - Upper semicontinuous decompositions of $E^{3}$, Ann. of Math. 65 (1957), 363-374.

6. R. Finney, Point-like, simplicial mappings of a 3-sphere, Canad. J. Math. 15 (1963), 591-604.

7. K. W. Kwun and F. Raymond, Almost acyclic maps of manifolds, Amer. J. Math. 86 (1964), 638-650.

UNIVERSITY OF UTAH AND

UNIVERSITY OF IOWA 